
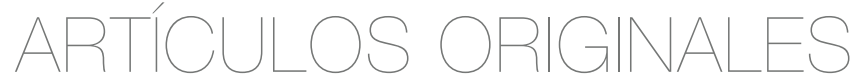

\title{
Beneficios contables y fiscales de los contribuyentes de negocios plataneros en México
}

\author{
GERMÁN MARTÍNEZ PRATS* \\ SERGIO CHÁVEZ NAVARRO**
}

* Doctor en Gobierno y Administración Pública. Universidad Juárez Autónoma de Tabasco, Villahermosa, México. E-mail: german.martinez@ujat.mx. ORCID: oooo-0001-6371-448X. Google Scholar: https://scholar.google.com/ citations?user=qbPIXmEAAAAJ\&hl=en.

** Magíster en Contaduría. Universidad Juárez Autónoma de Tabasco, Villahermosa, México. E-mail: sergio.chavez@ujat.mx. ORCID: 0000-0001-7582-3608. Google Scholar: http://scholar.google.com/citations?user=tplDKBMAAAAJ\&hl=es. 


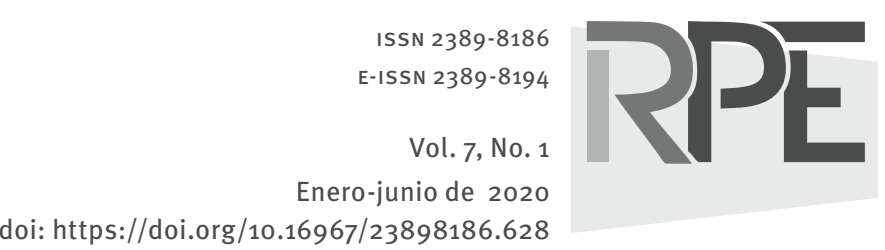

COMO CITAR ESTE ARTÍCULO

How to cite this article:

Martínez, G. y Chávez, S. (2020). Beneficios contables y fiscales de los contribuyentes de negocios plataneros en México. Revista Perspectiva Empresarial, 7(1), 61-71.

Recibido: 11 de enero de 2020

Aprobado: 30 de marzo de 2020
RESUMEN Un elevado sector de la sociedad en México se desarrolla principalmente con actividades agropecuarias que tienen una alta participación en el PIB, permitiendo que el país a la vez tenga cierta autonomía y suficiencia alimentaria. El impacto positivo del sector primario tiene como base las diversas contribuciones que realiza a factores tales como el mercado, la producción y las divisas. Al transcurrir el tiempo, y con la tecnificación suficiente, las actividades de este sector definen el éxito del entorno económico de las Naciones.

El presente texto tiene como objetivo describir los beneficios fiscales aplicables a los contribuyentes de negocios plataneros, los cuales se encuentran en el régimen de Actividades Agrícolas, Ganaderas, Silvícolas y Pesqueras -AGASPES-. El estudio tiene alcances descriptivos de tipo cualitativo, detallando las ventajas de aplicar los beneficios fiscales a los contribuyentes del sector primario al utilizar ejemplos con cifras estimadas. El tipo de investigación es documental, ya que para el análisis se utilizaron fuentes impresas y artículos consultados en línea relacionados con las leyes y disposiciones fiscales emitidas para aquellos contribuyentes del sector primario. En suma, se puede deducir que la aplicación de los beneficios fiscales son un aliciente al empresario del sector platanero que les permite tener un mayor flujo de efectivo.

PALABRAS CLAVE beneficios fiscales, sector primario, sector platanero, contabilidad.

\section{Accounting and tax benefits for taxpayers of plantain-growing businesses in Mexico}

ABSTRACT An important part of the Mexican society is developed mainly around agro farming activities which represent a high participation in the GDP, thereby allowing the country to have certain food autonomy and self-sufficiency. The positive impact of the primary sector is based on the diverse contributions it makes to factors such as the market, production and foreign exchange. Over time, and with enough technical advancement, the activities in this sector define the success of Nations' economic environment.

This paper aims at describing the tax benefits applicable to taxpayers from the plantain-growing businesses who are classified in the category of Agricultural, Cattle Raising, Forestry and Fishing Activities. The study has a qualitative descriptive scope, detailing the advantages of applying tax benefits to taxpayers from the primary sector by using examples with estimated figures. This research is of documentary type since for the analysis it used print sources and online articles related to tax laws and dispositions issued for those taxpayers from the primary sector. In sum, it can be deducted that the application of tax benefits is a business incentive for the plantain-growing sector businesspeople, which allows them to have greater cash flow.

KEY WORDS costs, accounting, management, prices and profitability. 


\section{Benefícios contábeis e fiscais dos contribuintes das empresas bananeiras no México}

RESUMO Um alto setor da sociedade no México é desenvolvido principalmente com atividades agrícolas com alta participação no PIB, permitindo ao país, ao mesmo tempo, ter alguma autonomia e suficiência alimentar. 0 impacto positivo do setor primário é baseado nas várias contribuições que faz a fatores como mercado, produção e câmbio. Com o passar do tempo, e com suficiente tecnificação, as atividades desse setor definem o sucesso do ambiente econômico das Nações.

O objetivo deste texto é descrever os benefícios fiscais aplicáveis aos contribuintes das empresas bananeiras, que se enquadram no regime de Atividades Agrícolas, Pecuárias, Silviculturais e de Pesca. 0 estudo possui um escopo descritivo qualitativo, detalhando as vantagens da aplicação de benefícios fiscais aos contribuintes no setor primário ao usar exemplos com valores estimados. 0 tipo de investigação é documental, uma vez que a análise utilizou fontes impressas e consultou artigos on-line relacionados às leis e disposições fiscais emitidas para os contribuintes do setor primário. Em suma, pode-se deduzir que a aplicação de benefícios fiscais é um incentivo ao empresário do setor de bananas que thes permite ter um maior fluxo de caixa.

PALAVRAS-CHAVE benefícios fiscais, setor primário, setor bananeiro, contabilidade. 


\section{Introducción}

El beneficio fiscal es una medida legislativa que dispone pagar menos impuestos por parte del contribuyente de manera legal. El Diccionario económico y fiscal tiene como sus características (i) que está sujeto a una serie de condiciones y (ii) que afecta de manera individual, no global. Es toda excepción al régimen general vigente del tributo que se articula con aplicación de técnica de exención y que supone una minoración de deuda tributaria; las exenciones pueden tener como objetivo incidir positivamente en el sistema económico o bien conseguir y aplicar una justicia tributaria; no obstante, si la exención se considera y aplica de forma técnica no es como tal un beneficio fiscal porque no persigue los objetivos citados (Durán, 2016).

A su vez tiene el carácter fiscal como ingreso y gasto público, la subvención es un dinero recaudado por la administración y destinado en la expansión y financiamiento de actividades convenientes y acordes con el interés general (de la Cruz y González, 2017).

En el caso de México, la Ley del Impuesto sobre la Renta -LISR- en su artículo 74 (fracción II y III) plantea:

Articulo 74. Deberán cumplir con sus obligaciones fiscales en materia del impuesto sobre la renta conforme al régimen establecido en el presente Capitulo, los siguientes contribuyentes:

[...] II. Las personas morales que se dediquen exclusivamente a actividades pesqueras, así como las sociedades cooperativas de producción que se dediquen exclusivamente a dichas actividades.

III. Las personas físicas que se dediquen exclusivamente a actividades agrícolas, ganaderas, silvícolas o pesqueras.

Se específica que de acuerdo a la actividad exclusiva de un empresario del sector platanero, en el régimen de Actividades Agrícolas, Ganaderas, Silvícolas y Pesqueras -AGASPES-, $\mathrm{y}$ tal como dispone la ley, sus ingresos totales deberán ser conformados por lo menos por el $90 \%$ de estas actividades. Al cumplir con este porcentaje marcado por la ley, los empresarios del sector platanero pueden acceder a los estímulos estipulados en las diversas disposiciones fiscales $\mathrm{y}$ a ciertas facilidades administrativas que les permite tener flexibilidad en sus operaciones contables.

En la misma fracción del artículo citado se establece que aquellos contribuyentes personas morales, conformados exclusivamente por personas físicas, pueden actuar como coordinados y cumplir con las obligaciones fiscales de cálculo y entero de impuestos por estos.

Los empresarios del sector platanero que opten por tomar los beneficios aplicados al título de los contribuyentes de AGASPES deberán estar en los supuestos ya mencionados y una vez que cumplan con los requisitos marcados por los diversos preceptos legales podrán acceder a los siguientes beneficios: (i) determinación de ingresos acumulables y deducciones autorizadas con base a flujo de efectivo; (ii) exención de ingresos y reducción de ISR, hasta ciertos montos; (iii) aplicación de tasa del 0 \% del IVA; (iv) aplicación de facilidades administrativas para efectos de comprobación y para el cálculo y retención de impuestos.

\section{Ingresos acumulables y deducciones autorizadas con bases a flujos de efectivos}

\author{
Para el doctrinante Luna (2018): \\ las personas morales que no realicen actividades \\ empresariales por cuenta de sus integrantes, deberán \\ determinar sus pagos provisionales en los términos \\ del artículo 106 de la LISR, aplicando al resultado \\ que obtenga la tasa del $30 \%$, es decir, el mecanismo \\ de personas físicas, pero aplicando la tasa del 30\%.
}

En este caso, el autor, en el análisis del marco jurídico aplicable a los contribuyentes del sector primario, establece que se aplica la determinación con base al mecanismo de personas físicas; pues estas acumulan sus ingresos y deducen sus gastos a través del flujo de efectivo (esto es, lo efectivamente cobrado o pagado). 
Los ingresos que se expresarán en las declaraciones mensuales y anuales para efectos de los pagos provisionales son los ingresos nominales y que provengan de enajenación de bienes y prestación de servicios de acuerdo a lo expresado en los artículos 14 y 17 de la LISR. Sin embargo se expresan los ingresos efectivamente cobrados o realizados y que puedan ser identificables en los estados de cuenta, aunque si se da una venta a crédito o se cuenta con el valor cuantificable de la venta se registra en el sistema de información contable para dar cumplimiento al postulado básico de devengación contable estipulado en la NIF A-2.

Las erogaciones podrán ser deducibles si se cumple lo estipulado en el artículo 27 de la LISR, a saber: (i) ser estrictamente indispensable; (ii) que las deducciones de inversiones se realicen conforme a la LISR; (iii) estar amparadas con un CFDI; (iv) utilizar medios bancarios cuando el monto de la erogación sea mayor a \$2,000.00 y donde deberá de contener el RFC del beneficiario del pago; (v) estar debidamente registradas en contabilidad y (vi) cumplir las disposiciones en materia de retenciones.

Para el costo de empresarios del sector platanero, al conformarse de activos biológicos que no sufren ningún tipo de cambio industrial, se maneja como si fuera actividades comerciales. Para ello el artículo 39 de la LISR señala que considerarán dentro de su costo los importes netos de mercancías (es decir, el importe original de adquisición de la mercancía menos los descuentos y devoluciones), así como el valor monetario de los gastos y las erogaciones relacionadas directamente con el desarrollo oportuno de las ventas de los bienes del contribuyente.

\section{Exención de ingresos}

El artículo 74 de la LISR, en su párrafo 21, menciona que las personas físicas no pagarán el impuesto sobre la renta por los ingresos provenientes de actividades agrícolas hasta por un monto de 40 veces el salario mínimo general elevado al año. En el caso de las personas morales que se dediquen exclusivamente a las actividades agrícolas, ganaderas, silvícolas o pesqueras no pagarán el impuesto sobre la renta por los ingresos provenientes de esas actividades hasta por un monto de 20 veces el salario mínimo general elevado al año y por cada uno de sus socios o asociados siempre que no exceda en su totalidad 200 veces el salario mínimo general elevado al año.

En este artículo se limita el monto a 200 salarios mínimos -que representa a diez socios o accionistas- para efectos de personas morales y tan solo es aplicable cuando la entidad obtenga ingresos en el ejercicio al equivalente a 423 veces el salario mínimo general elevado al año o menos, en caso contrario se pagará por el excedente el impuesto de la misma forma que para personas morales régimen general; con la diferencia de que se reducirá el impuesto determinado en un $30 \%$.

En el artículo 28, fracción II, se menciona que no serán deducibles los gastos e inversiones en la proporción que representen los ingresos exentos respecto del total de ingresos del contribuyente. Las deducciones serán supeditadas de acuerdo al monto de ingresos exentos que se aplique, considerando los efectos para el cálculo del IVA y lo establecido en el mencionado artículo.

Por ello el artículo 5 de la Ley del Impuesto al Valor Agregado -LIVA- indica que para que proceda el acreditamiento del impuesto debe corresponder a bienes, servicios o al uso o goce temporal de bienes que sean estrictamente indispensables para la realización de actividades distintas a la importación.

\section{Tasa del $0 \%$ de IVA para efectos de ingresos}

Los empresarios del sector platanero aplican la tasa del $0 \%$ en las actividades que le generaran ingresos, ya que como estipula el artículo 2-A de la LIVA se aplicará esa tasa cuando se enajenen animales y vegetales sin cambio industrial alguno.

El artículo 5 de la LIVA reafirma que para que una erogación sea acreditable debe ser estrictamente indispensable y cumplir con los requisitos de deducibilidad en los términos 
del artículo 27 de la LISR; para que con ello -y conforme lo estipula el artículo 6 de la LIVApueda solicitar su devolución, acreditamiento o compensación.

\section{Beneficios de la Resolución de facilidades administrativas}

\begin{abstract}
La regla 1.2., Resolución de facilidades administrativas para los sectores de contribuyentes -que en la misma se señalan-, estipula que los contribuyentes que desarrollan actividades de AGASPES podrán deducir gastos con documentación comprobatoria que cumplan con las fracciones I a la III y que no excedan el 10 $\%$ del total de los ingresos de la entidad; esto, sin exceder de $\$ 800,000.00$.
\end{abstract}

La regla 1.3., denota que los contribuyentes del sector primario que estén en el supuesto del artículo 74 de la LISR (como los empresarios plataneros) podrán optar a realizar sus pagos provisionales del ISR y del IVA de forma semestral. De la misma manera en la regla 1.8., se plantea que para efectos de las erogaciones que no excedan a $\$ 5,000.00$ se podrán pagar en efectivo sin necesidad de expedir cheques o realizar pagos con tarjeta de crédito o débito de propiedad del contribuyente. Por último, en la regla 1.10., se establece que se puede pagar en efectivo (sin necesidad de emisión de cheque o tarjeta de débito, crédito o transferencia bancaria) siempre y cuando no se exceda el $15 \%$ de los pagos efectuados por consumo de combustible para realizar su actividad.

\section{Metodología}

El presente texto tiene un enfoque cualitativo (Hernández, Fernández y Baptista, 2014) a través de la recolección de datos, lo que implica su análisis y sistematización; aunque vale la pena aclarar que no se utilizan datos cuantitativos para generar conclusiones, tan solo en la ejemplificación de los beneficios fiscales para la obtención de datos y resultados representativos y detallados.
El alcance de este estudio es descriptivo debido a que se señalan los beneficios fiscales que obtienen aquellos productores del sector platanero pertenecientes al régimen de AGASPES a través de la articulación con fuentes doctrinarias, lo que permitió basar ciertos conceptos o argumentos en diversas corrientes y sus autores; otra fuente de información fue la establecida en la norma por medio del análisis exegético de leyes y disposiciones fiscales emitidas para aquellos contribuyentes del sector primario (tales como la LISR y el Código Fiscal de la Federación -CFF-), estos textos legales otorgan el sentido real de la disposición sin que haya discrepancia alguna.

Por último, para la recolección y revisión de datos se utilizó la investigación documental de los temas establecidos y el análisis respectivo de los hallazgos encontrados y que son objeto de este estudio. El análisis se basa en las posiciones teóricas de Huberman y Miles (2000) y Salgado (2007), quienes desarrollaron tres subprocesos que se vinculan entre sí: la reducción de datos (selección y condensación); la presentación de datos (presentaciones concentradas en resúmenes) y la elaboración y verificación de conclusiones (triangulación de la información).

\section{Mercado platanero}

La FAO (2014) menciona dos hechos importantes en el sector platanero a nivel internacional, los cuales son: (i) la fusión de la empresa Chiquita Brands, la mayor empresa de comercio bananero en el mundo y con presencia dominante en el mercado de EEUU, y Fyffes uno de los principales proveedores del mercado europeo; (ii) se fortaleció el trabajo de la FAO con los gobiernos para brindar apoyo a los productores de plátano, informándolos y preparándolos con la aplicación de buenas prácticas de cultivo y gestión de sus empresas.

Para saber el impacto que tiene el cultivo de plátano en el sector nacional se hizo una consulta al Servicio de Información Agroalimentaria y Pesquera -SIAP-, los resultados obtenidos se muestran en la tabla 1. 
Tabla 1. Valor de producción de los cultivos en México

\begin{tabular}{|c|c|c|c|c|c|}
\hline Producto & $\begin{array}{c}\text { Superficie } \\
\text { sembrada (ha) }\end{array}$ & $\begin{array}{c}\text { Superficie } \\
\text { siniestrada (ha) }\end{array}$ & $\begin{array}{c}\text { Superficie } \\
\text { cosechada (ha) }\end{array}$ & Producción (ton) & $\begin{array}{c}\text { Rendimiento } \\
\text { (ton/ha) }\end{array}$ \\
\hline Caña de azúcar & 822,897 & & 745,590 & $56,007,311$ & 75,118 \\
\hline Alfalfa verde & 388,448 & 291 & 385,461 & $33,893,357$ & 87,929 \\
\hline Rosa (gruesa) & 1,717 & & 1,696 & $8,774,381$ & $5,174.64$ \\
\hline Naranja & 340,586 & 24 & 324,246 & $4,670,259$ & 14,403 \\
\hline Limón & 198,310 & 31 & 171,652 & $2,520,797$ & 14,686 \\
\hline Plátano & 80,309 & 1,155 & 77,229 & $2,203,069$ & 28,527 \\
\hline Aguacate & 220,334 & 43 & 194,400 & $1,997,629$ & 10,276 \\
\hline Mango & 201,423 & 1,207 & 188,575 & $1,958,132$ & 10,384 \\
\hline Agave & 105,690 & 185 & 22,055 & $1,682,148$ & 76,27 \\
\hline Gerbera (gruesa) & 116 & & 112 & $1,313,958$ & $11,679.63$ \\
\hline Papaya & 19,114 & 16 & 16,684 & 964,702 & 57,82 \\
\hline Piña & 42,907 & & 20,010 & 945,002 & 47,227 \\
\hline Café cereza & 717,388 & 3,446 & 641,478 & 838,997 & 1,308 \\
\hline Nopalitos & 12,718 & 332 & 12,033 & 828,971 & 68,894 \\
\hline Manzana & 57,770 & 26 & 53,691 & 714,462 & 13,307 \\
\hline Tuna & 47,984 & 377 & 45,370 & 469,844 & 10,356 \\
\hline Toronja (pomelo) & 20,127 & 9 & 18,707 & 451,814 & 24,152 \\
\hline Uva & 33,702 & 75 & 30,093 & 418,511 & 13,907 \\
\hline Guayaba & 22,555 & & 22,165 & 323,667 & 14,603 \\
\hline Zarzamora & 12,433 & & 12,179 & 266,764 & 21,904 \\
\hline Esparrago & 27,409 & 136 & 25,828 & 240,983 & 9,33 \\
\hline Copra & 128,058 & & 124,542 & 231,174 & 1,856 \\
\hline Maguey pulquero & 9,093 & & 3,980 & 217,691 & 54,691 \\
\hline Durazno & 31,551 & 2,430 & 25,602 & 161,867 & 6,322 \\
\hline Nuez & 123,266 & & 90,079 & 150,349 & 1,669 \\
\hline Frambuesa & 5,639 & & 5,559 & 109,334 & 19,667 \\
\hline Cacao & 59,920 & & 58,758 & 27,326 & 0,465 \\
\hline Total & $3,735,712$ & 9,783 & $3,321,929$ & & \\
\hline
\end{tabular}

Fuente: elaboración propia por parte de los autores.

En la tabla 1 se puede observar que a nivel nacional el plátano se encuentra en el sexto lugar en orden ascendente a descendente, tomando el valor de producción en toneladas; por tanto, su cultivo es relevante debido a que se encuentra entre los diez primeros a nivel nacional del tipo perenne.

De acuerdo con la Secretaría de Agricultura, Ganadería, Desarrollo Cultural -SAGARPA-, para 2015, hay una serie de hechos y datos relevantes del mercado platanero en el país:
1. A nivel nacional entre 2013 y 2015 México tuvo una ocupación de sembradíos de hectáreas de plátano de 72000 a 75000 , generando una producción anual durante ese período de 2,2 millones de toneladas con un rendimiento de 29 toneladas por hectárea.

2. La producción de plátano se destina en un $92 \%$ a consumo interno y un $8 \%$ a exportación.

3. Las principales locaciones del país donde se concentra la producción de plátano son las 
En la tabla 1 se puede observar que a nivel nacional el plátano se encuentra en el sexto lugar en orden ascendente a descendente, tomando el valor de producción en toneladas; por tanto, su cultivo es relevante debido a que se encuentra entre los diez primeros a nivel nacional del tipo perenne.

De acuerdo con la Secretaría de Agricultura, Ganadería, Desarrollo Cultural -SAGARPA-, para 2015, hay una serie de hechos y datos relevantes del mercado platanero en el país:

1. A nivel nacional entre 2013 y 2015 México tuvo una ocupación de sembradíos de hectáreas de plátano de 72000 a 75000, generando una producción anual durante ese período de 2,2 millones de toneladas con un rendimiento de 29 toneladas por hectárea.

2. La producción de plátano se destina en un $92 \%$ a consumo interno y un 8 \% a exportación.

3. Las principales locaciones del país donde se concentra la producción de plátano son las regiones costeras del Golfo de México y el océano Pacífico; de las cuales lideran los Estados de Chiapas, Tabasco y Veracruz.

4. Para la fecha, Chiapas y Veracruz concentraban el $51 \%$ de la superficie cosechada del país; mientras que Tabasco solo contaba con el $14 \%$ de la superficie en ese período de tiempo.

5. Las variedades más importantes de plátano en orden de hectáreas sembradas son: primero el plátano enano gigante con una superficie cosechada de 29,629 hectáreas y una producción anual de 1,32 millones de toneladas; luego le sigue la variedad de plátano macho con una superficie cosechada de 17,830 hectáreas y una producción anual de 314,914 toneladas; finalmente se tienen las variedades dominico, tabasco, criollo y valery.

6. Para comercializar el plátano los grandes productores en México tienen integrado el proceso de comercialización, pues tienen bodegas en la Central de Abastos de Iztapalapa en Ciudad de México, estimándose que el $80 \%$ de la producción de los principales Estados productores se concentra en esta localización.
7. Para comercializar el plátano los medianos productores se basan en los comerciantes mayoristas mediante comisionistas. Los pequeños productores y ejidatarios recurren al acopiador local, luego se pasa al comisionista en la Central de Abasto y este último al comerciante mayorista.

8. Para la comercialización en el mercado internacional se distinguen dos canales: (i) los productores-comerciantes grandes, que cuentan con volúmenes importantes, tienen la posibilidad de prometer su producto a las empresas transnacionales; (ii) los productores medianos a través de empresas transnacionales, pero con menor precio que el gran productor.

9. Las Normas Oficiales Mexicanas -NOMaplicables al cultivo del banano-plátano, de forma enunciativa, son la NOM-010-FITO-1995 y la NMX-FF-029-1995-SCFI. La norma del Codex Alimentarius para el banano-plátano es la denominada CODEX STAN 205-1997.

10. Los procedimientos de certificación son voluntarios y entre ellos se destacan: Comercio Justo (FLO), Rainforest Alliance, SA8000, EurepGAP, ISO 14001, México Calidad Suprema, SQF (Safe Quality Food), Sistema de Reducción de Riesgos de Contaminación.

\section{Contribuyentes del sector primario}

Los contribuyentes que realizan actividades del sector primario (esto es, agricultura, ganadería, pesca y silvicultura) han tenido por más de tres décadas beneficios relacionados directamente con los ingresos obtenidos por esta actividad, particularmente relacionados con la tributación al ISR (Gallegos, 2015).

Luna (2018) hace mención de la evolución del tratamiento de los contribuyentes del sector primario, a saber: en 1991 se crea el régimen simplificado y la Resolución de facilidades administrativas; a partir de 1998 el régimen simplificado abarca exclusivamente las actividades del sector primario y autotransporte (incluyendo personas físicas y morales); en 2002 
se excluye dentro de este régimen a las personas físicas (consideradas solo como integrantes de las personas morales que tributan en este título); de 2008 a 2013 las diferencias entre las personas físicas y morales en cuanto a los beneficios fiscales del régimen de actividades relacionadas al sector primario son sobre operatividad y las contribuciones al ISR, al impuesto empresarial a tasa única -IETU-y de inversión extranjera directa -IDE-; desde 2014 se originó un título particular (el Régimen Fiscal de las Actividades Agropecuarias) que hasta 2018 ha tenido transformaciones en materia de fiscalización y rendición de cuentas por parte de los contribuyentes.

El tratamiento especial del régimen AGASPES, con entrada en vigor en 2014, tiene como justificación: (i) el apoyo a la autosuficiencia alimentaria para México y (ii) la adaptación al entorno operativo y de negocios de las actividades relacionadas al sector primario (Gallegos, 2015).

Para Brown (2013) el crecimiento del sector agropecuario es reforzado por inversiones en materia de investigación y tecnificación de la agricultura, así como el apoyo de políticas gubernamentales a este sector.

De acuerdo con el Congreso de la Unión, en el CFF en su artículo 16, se definen las actividades del sector primario del régimen AGASPES de la siguiente manera: las actividades agrícolas son aquellas que comprenden las actividades de siembra, cultivo y cosecha que no hayan sido objeto de transformación industrial; las actividades ganaderas son las consistentes en la cría y engorda de animales y que no hayan sufrido algún tipo de transformación que altere su naturaleza primordial; las actividades pesqueras incluyen la cría y reproducción de toda clase de especies marinas, sin que estas hayan sufrido tratamiento operativo.

\section{Resultados y discusión}

Se tiene como supuesto que los márgenes de utilidad de este sector oscilan entre un 15 y 20 $\%$ de utilidad bruta o base para impuestos; para efectos de este ejemplo se tomará su promedio, es decir, de 17,5\%. Este porcentaje se adicionará a la cantidad obtenida de ingresos exentos para sacar el factor al que hace referencia el artículo 28, fracción II, de la LISR.

Una vez que se han establecido de manera general los beneficios dados por los mismos preceptos legales y las obligaciones que hay que cumplir, en la tabla 2, se analizará el cálculo del ISR para las personas físicas y morales.

Tabla 2. Cálculo ISR persona física y persona moral

\begin{tabular}{lllll}
\hline \multicolumn{5}{c}{ Cálculo sector primario } \\
\hline & Persona física & $\$ 1,155,000.00$ & Persona moral & $\$ 4,500,000.00$ \\
\hline- & Ingresos exentos & $\$ 1,00,000.00$ & Ingresos exentos & $\$ 4,000,000.00$ \\
\hline- & Gastos en proporción ingresos exentos & $\$ 128,000.00$ & $\begin{array}{l}\text { Gastos en proporción ingresos } \\
\text { exentos }\end{array}$ & $\$ 10,000.00$ \\
\hline$=$ & Utilidad fiscal & $\$ 27,000.00$ & Utilidad fiscal & $\$ 490,000.00$ \\
\hline$*$ & ISR 40 \% & $\$ 10,800.00$ & ISR 40 \% & $\$ 181,403.83$ \\
\hline$=$ & ISR a pagar & $\$ 9,000.00$ & ISR a pagar & $\$ 308,596.17$ \\
\hline
\end{tabular}

Fuente: elaboración propia por parte de los autores. 
Se observa en la tabla 2 que la reducción al ISR es uno de los beneficios fiscales más atractivos de este sector (Rosiles et al., 2017); de manera paralela la devolución del IVA de acuerdo a las reglas que marca la ley, que norman el tributo del mencionado impuesto; de ahí que el $90 \%$ de los ingresos totales deben referirse a estas actividades, excepto por los ingresos derivados de las enajenaciones de activos fijos y terrenos (Cavazos, 2014).

Mediante el análisis de las cifras que ejemplifican los beneficios fiscales se puede deducir que la aplicación de estos son un aliciente al empresario del sector platanero, los cuales le permiten tener un mayor flujo de efectivo en comparación con aquellos empresarios que no tributen en actividades exclusivamente del sector primario como marca la LISR.

Sin embargo se recomienda cumplir cabalmente con las obligaciones fiscales (Rodríguez, 2019) que se han mencionado a lo largo de esta investigación, ya que puede generar una carga administrativa o financiera para poder cumplir con las auditorías electrónicas o de escritorio aplicadas a la entidad.

Estos beneficios, de la mano de políticas públicas centradas en apoyar al campo y al sector agropecuario, tienen como fin intrínseco disminuir las tendencias que marcan de manera general las actividades del sector primario (Martínez, Salgado y Vázquez, 2017) y que han llevado a presentar una reducción de su inversión para 2018. De igual manera llama la atención que el beneficio al estrato económico más bajo (régimen para pequeños productores del sector primario) ha decrecido a una tasa media anual real de 9,2\%; lo anterior, sugiere que el diseño de los estímulos y las características del mercado fomentan que estos productores no aprovechen los beneficios otorgados para el sector.

\section{Conclusión}

En la práctica profesional se observa que este tipo de empresas aplica a los beneficios fiscales ya mencionados, pero en algunas situaciones no cumplen con todas las disposiciones que marcan los preceptos legales y aplicables al sector primario. Se recomienda para las solicitudes de devoluciones de forma inmediata o de manera tradicional que se verifique que los registros contables cumplan con lo estipulado en las normas y preceptos que rigen la contaduría pública. Entre ellos, lo más importante es el de llevar la contabilidad de la siguiente manera:

1. La entidad debe realizar los registros contables conforme a las disposiciones fiscales que se mencionan en el reglamento de la LISR, el CFF y su reglamento, formulando el estado de posición financiero y realizar la valuación y control de sus inventarios de acuerdo a lo estipulado por el artículo 76 de la misma LISR y el artículo 32 de la LIVA.

2. La contabilidad se realizará en sistemas de información contables electrónicos que tal como marca el artículo 28 del CFF se integrarán por las pólizas contables y su documentación soporte generada por la entidad ya sea de índole administrativa o fiscal, los estados de cuentas bancarios y los demás medios electrónicos que sirvan de apoyo a los registros contables y con información económica contable.

3. El artículo 33 del Reglamento del Código Fiscal de la Federación -RCFF- detalla los documentos que también formarán parte del sistema de información contable (anexo a lo estipulado al 28 del CFF) entre los que destacan las declaraciones provisionales, informativas o definitivas, el catálogo de cuentas, las conciliaciones bancarias y los contratos de los trabajadores.

4. Retomando el artículo 33 del RCFF, este menciona que los registros contables deben revelar suficientemente las operaciones en que incurra la entidad y a más tardar dentro de los 
cinco días hábiles a su realización; lo anterior, se relaciona con los CFDI que dieron origen a dicha operación y con ello generar los estados financieros básicos y sus notas.

Esto con la finalidad de que si la autoridad solicita más información para corroborar las operaciones de la entidad o procede con la fiscalización electrónica, el empresario del sector platanero no presente ninguna dificultad al momento de acceder a la devolución de impuestos.

\section{Referencias}

Brown, W. (2013). El papel de la agricultura en la reducción de la pobreza. Revista Mexicana de Agronegocios, 32, 166-178.

Cavazos, M. (2014). Aspectos fiscales importantes del régimen agropecuario. Fiscoactualidades, 21, 3-16.

de la Cruz, M. y González, M.Á. (2018). Los beneficios fiscales en la doctrina. Revista Instituto Colombiano de Derecho Tributario, 66, 61-102.

Durán, J. (2016). Los beneficios fiscales en el sistema tributario estatal español (tesis de grado). Facultad de Economía y Empresa, Universidad de la Coruña, Coruña, España.

FAO. (2014). Banana Market Review 2013-2014. Roma, Italia: FAO.

Gallegos, J. (2015). Reducción del ISR para contribuyentes dedicados a realizar actividades del sector primario. Fiscoactualidades, 4, 3-18.

Hernández, R., Fernández, C. y Baptista, P. (2014). Metodología de la investigación. Ciudad de México, México: McGraw-Hill.

Huberman, A. y Miles, M. (2000). Métodos para el manejo y el análisis de datos. En Denman, C. y Haro, J.A. (Coord.), Por los rincones. Antología de métodos cualitativos en la investigación social (pp. 253-301). Sonora, México: El Colegio de Sonora.

Luna, A. (2018). Régimen de incorporación fiscal 2018. Ciudad de México, México: Ediciones Fiscales ISEF.

Martínez, A., Salgado, A. y Vázquez, S. (2017). Tendencias recientes del sector primario en México. Recuperado de https://www.bbvaresearch.com/wp-content/ uploads/2017/03/170316_Mexico_Agropecuario. pdf.

Rosiles, L. M. et al. (2017). Reforma fiscal mexicana 2014 estrategia que fomenta la formalidad empresarial, generación de empleo, financiamiento, crecimiento y competitividad. Revista Global de Negocios, 5(3), 13-31.

Rodríguez, G. (2019). Obligaciones fiscales. Boletín Mexicano de Derecho Comparado. Ciudad de México, México: UNAM.

Salgado, A. (2007). Investigación cualitativa: diseños, evaluación del rigor metodológico y retos. LIBERABIT, 13(13), 71-78. 